\section{Damping of Acoustic Waves in Dilute Polymer Solutions}

\section{Y. Tsori and P.-G. de Gennes}

Physique de la Matière Condensée, Collège de

+ France, Paris, France

1. Introduction. The shear viscosity of dilute polymer solutions $\eta_{S}(\omega)$ has been studied in the past in experiments and models and analyzed in a classic book by Ferry. 1 The standard view is that each polymer coil has a sequence of modes discussed by Zimm ${ }^{2}$ which are due to hydrodyNnamic interactions between the monomers; the viscosity $\eta_{S}$ then appears as a weighted sum of Lorentzian responses from these modes. For qualitative purposes, at frequencies which are not too high, the first mode dominates, as noticed very searly by Peterlin, and $\eta_{S}$ can be reduced to a simple form,

$$
\eta_{S}(\omega)=\alpha_{s} \nu k_{B} T \frac{\tau_{z}}{1+w^{2} \tau_{z}^{2}}
$$

Here $\alpha_{s}$ is a coefficient of order unity, $\nu$ is the number of polymer coils per unit volume, $k_{B} T$ is the thermal energy, $\omega$ is the angular frequency, and $\tau_{z}$ is the Zimm relaxation time, with the scaling structure $\underline{3}$

$$
\tau_{z} \simeq \frac{\eta R_{F}^{3}}{k_{B} T}
$$

Here $\eta$ is the solvent shear viscosity, and $R_{F}$ is the coil size, which we will estimate for good solvents following Flory's $\operatorname{approach} 3$ [4

$$
R_{F}^{5}=a^{5}(1-2 \chi) N^{3}
$$

where $a$ is the monomer size, $N$ is the number of monomers in a chain, and $\chi$ is the Flory interaction parameter. 4

Our aim in the present note is to discuss the longitudinal viscosity $\eta_{L}(\omega)$ which may be measured via attenuation of ultrasound $=$ this implies that we now consider the solution as a (weakly) compressible fluid. For incompressible fluids, -symmetry imposes that $\eta_{L}=\frac{4}{3} \eta_{S}$. But here we must write

$$
\eta_{L}=\frac{4}{3} \eta_{s}+\eta_{p}
$$

where $\eta_{P}$ includes specific effects of density changes, or (equivalently) of the applied pressure $p$ (see section 3).

Our basic idea is that $p$ modifies the $\chi$ parameter, and thus modifies $R_{F}$, as indicated in eq 3 . We construct a crude model for the resulting dynamics, assuming an analogue of Peterlin's approach, with a single mode relaxation time $\tau_{z}$.

The equilibrium results are presented in section 2 , and the dynamics is schematized in section 3. The surprising conclusion is that $\eta_{P}$ is at least comparable to $\eta_{S}$, even in liquids which are not very compressible; this is discussed in section 4. All the exploration is made only at the level of scaling laws.
2. Basic Couplings Between Coil Size and Pressure. We start from a coil in good solvent, with a size described by the Flory expression, eq 3 . We then raise the pressure by an amount $p$; the equilibrium size shifts by a certain amount $\delta R$. We can think of (at least) two contributions to $\delta R$.

The first contribution, $\delta R_{K}$, is due to a change in Kuhn length, which may be written in the form

$$
\frac{\delta R_{K}}{R_{K}}=\alpha_{K} \frac{p}{K}
$$

where $K \simeq 10^{3} \mathrm{MPa}$ is the elastic modulus of the solvent, $p / K$ measures the solvent contraction, and $\alpha_{K}$ is a number which is expected to be of order unity (and which may be positive or negative).

The second contribution, $\delta R \chi$, is the consequence of a change $\delta \chi$ in the Flory parameter $\chi$,

$$
\delta \chi=\alpha_{\chi} \frac{p}{K}
$$

Again we expect $\left|\alpha_{\chi}\right|$ to be of order unity. [5] 6 The resulting shift in size is obtained by differentiating eq 3

$$
\frac{\delta R_{\chi}}{R_{F}}=-\frac{2}{5} \frac{\delta \chi}{1-2 \chi}
$$

Equations 3 and $[7$ hold provided that we are in a strong swelling regime, i.e., that the perturbation parameter $\zeta$ describing the excluded volume is small. 7 The scaling structure of $\zeta$ is $\zeta=N^{1 / 2}(1-2 \chi)$ and thus eq 7 assumes that

$$
1-2 \chi>N^{-1 / 2}
$$

We arrive now to an interesting point. When the pressure $p$ is applied, the solvent is contracted $=$ all linear dimensions are reduced by a factor $1-p / 3 K$. If the relative shift of $\delta R$ was just equal to $-p / 3 K$, the whole system would be just modified by an affine deformation, and no relaxation process would be involved. Thus we must define an effective chain deformation $\delta R_{\text {eff }}$, where the affine contribution has been taken out. Collecting all these results we arrive at

$$
\begin{aligned}
\frac{\delta R_{\mathrm{eff}}}{R_{F}} & =\alpha \frac{p}{K} \\
\alpha & =\alpha_{K}-\frac{2}{5} \frac{\alpha_{\chi}}{1-2 \chi}-\frac{1}{3}
\end{aligned}
$$

However, we immediately note that the $\alpha_{\chi}$ term will dominate if

$$
1 \gg 1-2 \chi \gg N^{-1 / 2}
$$

3. The Longitudinal Viscosity. For a compression wave in a liquid, individual molecules can move parallel or perpendicular to the direction of wave propagation. The existence of these two modes means that energy dissipation 
depends on two independent quantities, hence there are two fundamental viscosities. The longitudinal compression mode brings about a term $\mathbf{d i v} \cdot \mathbf{v}$ in the Navier-Stokes equation, a term which is absent in the more usual case of an incompressible liquid. In the linear theory employed here, it is sufficient to consider only monochromatic waves; the superposition of many modes is trivial. For such a longitudinal wave in the $x$-direction, the velocity of molecules is given by

$$
v=v_{x}=v_{0} \cos (k x+w t)
$$

where $\omega$ is the frequency, $c=\omega / k \approx 1500 \mathrm{~m} / \mathrm{s}$ is the wave velocity $\left(c \gg v_{0}\right)$, and $k$ is the wavevector. For frequencies up to megahertz, the sound wavelength is much larger than the coil size. Each chain then feels a time-varying pressure $p=p_{0} e^{i \omega t}$, with $v_{0} / c=p_{0} / K$. It can be shown 8 that the energy dissipation per unit volume is given by

$$
\begin{aligned}
T \dot{S} & =\frac{1}{2} v_{0}^{2} k^{2}\left(\frac{4}{3} \eta_{S}+\eta_{P}\right) \\
& =\frac{1}{2}\left(\frac{\omega p_{0}}{K}\right)^{2} \eta_{L}(\omega)
\end{aligned}
$$

where we have defined $\eta_{L}(\omega) \equiv \frac{4}{3} \eta_{S}(\omega)+\eta_{P}(\omega)$. $\eta_{P}$ is comparable to $\eta_{S}$ and always present in a longitudinal wave. However, in the presence of internal slow relaxation processes (e.g., slow chemical reactions) it is enhanced. In the following, our aim is to find the expression for $\eta_{L}$ relevant to polymer solutions.

It is important to realize that, for an acoustic wave, the coefficient $\alpha$ is renormalized by a thermal effect. The wave is adiabatic with temperature modulation

$$
\delta T=q p
$$

where $q=\left(T / C_{p} V\right)(\partial V / \partial T)_{p}$ and $V$ is the volume.

For instance, the Kuhn length term $\alpha_{K}$ is renormalized to a new value

$$
\tilde{\alpha}_{K}=\alpha_{K}+\frac{q}{R_{K}} \frac{d R_{K}}{d T}
$$

We expect a similar renormalization for $\alpha_{\chi}$. This renormalization should not affect the scaling law which we postulated in section 2 .

As we saw in the previous section, the equilibrium size of the coil is modified from its Flory radius $R_{F}$ by an amount $\alpha p R_{F} / K$ proportional to the external pressure. The polymer, characterized by a "response time" $\tau_{z}$, does not follow this size instantaneously, but rather with a certain time lag. This time lag leads to dissipation. Thus we may denote $\delta R$ as the instantaneous deviation of the coil size from the Flory size, and write

$$
\delta \dot{R}=\frac{1}{\tau_{z}}\left(\alpha \frac{p}{K} R_{F}-\delta R\right)
$$

The solution is straightforward: $\delta R=\delta R_{0} e^{i \omega t}$ and

$$
\delta R_{0}=\frac{\alpha}{K} \frac{p_{0}}{1+i \omega \tau_{z}} R_{F}
$$

An equivalent formulation is to imagine an oscillating spring in a viscous media characterized by a friction constant $\xi$

$$
\xi \delta \dot{R}=\frac{k_{B} T}{R_{0}^{2}}\left(\alpha \frac{p}{K} R_{F}-\delta R\right)
$$

Here the spring constant is $k_{B} T / R_{0}^{2}$. Hence, we identify the friction constant as $\xi=\tau_{z} k_{B} T / R_{0}^{2}$, and the total dissipation per unit volume is then

$$
\begin{aligned}
T \dot{S} & =\frac{1}{2} \nu \xi|\delta \dot{R}|^{2} \\
& =\frac{1}{2} \nu \tau_{z} \frac{k_{B} T}{R_{0}^{2}}\left(\frac{\alpha p_{0}}{K}\right)^{2} R_{F}^{2} \frac{\omega^{2}}{1+\omega^{2} \tau_{z}^{2}} \\
& \simeq \frac{1}{2} \nu \tau_{z} k_{B} T\left(\frac{\omega p_{0}}{K}\right)^{2} \frac{1}{(1-2 \chi)^{2}} \frac{1}{1+\omega^{2} \tau_{z}^{2}}
\end{aligned}
$$

In the last line we assumed that $\alpha$ is dominated by the $\alpha_{\chi}$ term (eq [10), and that $\alpha_{\chi}$ is of order unity.

Comparison with eq 14 leads to the identification of $\eta_{L}$ as

$$
\eta_{L}(\omega)=\nu \tau_{z} k_{B} T \frac{1}{(1-2 \chi)^{2}} \frac{1}{1+\omega^{2} \tau_{z}^{2}}
$$

This expression is identical in form to the one-mode expression for the shear viscosity $\eta_{S}$ in polymer solutions (eq 1), except for the $(1-2 \chi)^{-2}$ prefactor, which can be large in the vicinity of the $\Theta$ point.

How is the wave damped? In the above we have assumed that the wavevector $k$ is purely real. In fact, damping is described by an imaginary part of $k$ given in

$$
k=\frac{\omega}{c}+i \frac{1}{2 \rho c^{3}} \eta_{L} \omega^{2}
$$

with the imaginary part much smaller than the real one.

4. Conclusions. (1) We expect a specific (pressure induced) longitudinal viscosity $\eta_{L}(\omega)$ in polymer solutions. The pressure contribution should be most visible near the $\Theta$ point, with an enhancement factor

$$
f=\left(\frac{1}{1-2 \chi}\right)^{2} \sim\left(\frac{\Theta}{T-\Theta}\right)^{2}
$$

(provided that $f$ remains smaller than $N$ ). If we go below the $\Theta$ point, the pressure wave modulates a phase separation $=$ there is another dynamics to consider (this was pointed out to us by a referee). (2) We discussed the frequency dependence of $\eta_{P}$ in a "one-mode approximation". For the shear viscosity, the restriction to one mode is acceptable when $\omega \tau_{z}<1$. But the one-mode approximation could be worse for our case. We can look at the related problem of chain collapse under a temperature jump (from above $\Theta$ to below it). The early stages of collapse involve "local clumps" along the chain $=$ this is rather different from the standard 
deformation in shear. (3) Our main conclusion is that $\eta_{P}(\omega)$ may be observable in certain dilute polymer solutions. Can we expect this idea to hold for polymer melts? In a melt, the coils are ideal (as first understood by Flory 4 ) and the $\chi$ parameter becomes irrelevant. But we may still have a small effect of the pressure on the Kuhn length. (4) The pressure dependence of $\chi$ may also be of interest in block copolymer melts. Consider, for instance, a lamellar phase $=$ a change in $\chi$ leads to a change in the interfacial area per chain and to chain extension. However, in the strong segregation limit the lamellar period scales as $\sim N^{2 / 3} \chi^{1 / 6}$ and depends only weakly on the $\chi$ parameter.

\section{References}

[1] Ferry, J. D. Viscoelastic Properties of Polymers, 3rd ed.; Wiley-Interscience: New York, 1980.

[2] Zimm, B. H. J. Chem. Phys. 1956, 24, 269.

[3] de Gennes, P. G. , Scaling Concepts in Polymer Physics; Cornell University Press: Ithaca, NY, 1979.

[4] Flory, P. J. Principles of Polymer Chemistry; Cornell University Press: Ithaca, NY, 1971.

[5] Janssen, S.; Schwahn, D.; Mortensen, K.; Springer, T. Macromolecules 1993, 26, 5587. Schwahn, D.; Frielinghaus, H.; Mortensen, K.; Almadal, K. Phys. Rev. Lett. 1996, 77, 3153 .

[6] Janssen, J.; Schwahn, d.; Springer, T.; Mortensen, K. Macromolecules 1995, 28, 2555.

[7] Yamakawa, H. Modern Theory of Polymer Solutions; Harper and Row, NY, 1971.

[8] Landau, L. D.; Lifshitz, E. M. Fluid Mechanics; Pergamon: Oxford, 1979. 